\title{
Repeat Operation for Pseudoaneurysm of Left Ventricular Outflow in a Patient with Concomitant Takayasu's Arteritis and Marfan Syndrome
}

\author{
Satoshi Yamashiro, MD, PhD, Yukio Kuniyoshi, MD, PhD, Katsuya Arakaki, MD, Takaaki Nagano, MD, \\ and Yuya Kise, MD
}

\begin{abstract}
We describe concomitant Marfan syndrome and Takayasu's arteritis complicating a pseudoaneurysm of the left ventricular outflow that developed after aortic root reconstruction. A patient was admitted with a high fever four months after initial root reconstruction that included valve sparing (reimplantation) as well as coronary artery reconstruction using a Carrel's button technique. Computed tomography revealed a pseudoaneurysm at the posterior side of the aortic root. We applied a modified Bentall procedure including coronary artery reconstruction using the Piehler technique. Pathological assessment of a specimen of the aorta revealed no central medial necrosis, but significant lymphocytic infiltration and thick fibrous adventitia indicating Takayasu's arteritis. This case was unique in terms of having simultaneous Takayasu's arteritis and cardiovascular manifestations of Marfan syndrome that were surgically treated.
\end{abstract}

Keywords: chronic inflammation, aorta, stenosis, connective tissue disease

$\mathrm{T}$ akayasu's arteritis is chronic inflammation of the aorta, its branches, and the pulmonary artery that leads to stenosis/occlusion or dilation. Cardiovascular complications such as aortic valve regurgitation and aneurysmal dilation of the aortic root can be fatal. The most serious complication after root reconstruction for Takayasu's arteritis is dehiscence of the anastomotic site. ${ }^{1-4)}$ We describe a patient with Takayasu's arteritis associated with Marfan syndrome who underwent valve sparing root reconstruction for annulo-aortic ectasia with aortic regurgitation, but who required a repeated operation to treat a pseudoaneurysm five months later.

Thoracic and Cardiovascular Surgery Division, Ryukyu University Hospital, Okinawa, Japan

Received: March 2, 2011; Accepted: August 8, 2011 Correspondence author: Satoshi Yamashiro, MD, PhD. Thoracic and Cardiovascular Surgery Division, Ryukyu University Hospital, 207 Uehara, Nishihara, Okinawa 903-0215, Japan Tel: +81-98-895-1168; Fax: +81-98-895-1422

E-mail: y3104@med.u-ryukyu.ac.jp

\section{CASE Report}

A 19 year-old man with annulo-aortic ectasia, accompanied by an aneurysm of the ascending aorta and aortic arch and Marfan syndrome, had undergone root reconstruction and total arch replacement using the elephant trunk method. Preoperative computed tomography (CT) identified aneurysmal dilation from the Sinus of Valsalva to the arch aorta, and stenosis of the left subclavian artery (Fig. 1A). Ultrasound echocardiography revealed mild aortic valve regurgitation. The initial root reconstruction included valve sparing (David II) and coronary artery reconstruction using a Carrel's button technique. He had also undergone total arch replacement using the elephant trunk method with simultaneous reconstruction of the left subclavian artery (Fig. 1B). The postoperative course of this patient was unusual. A high fever persisted despite the absence of an obvious, infectious focus, according to pathological and bacterial analyses. The serum concentration of C-reactive protein (CRP) remained elevated despite negative bacterial cultures. Nineteen days after the initial operation, a pseudoaneurysm that ruptured in the previously cannulated femoral artery was repaired using a 

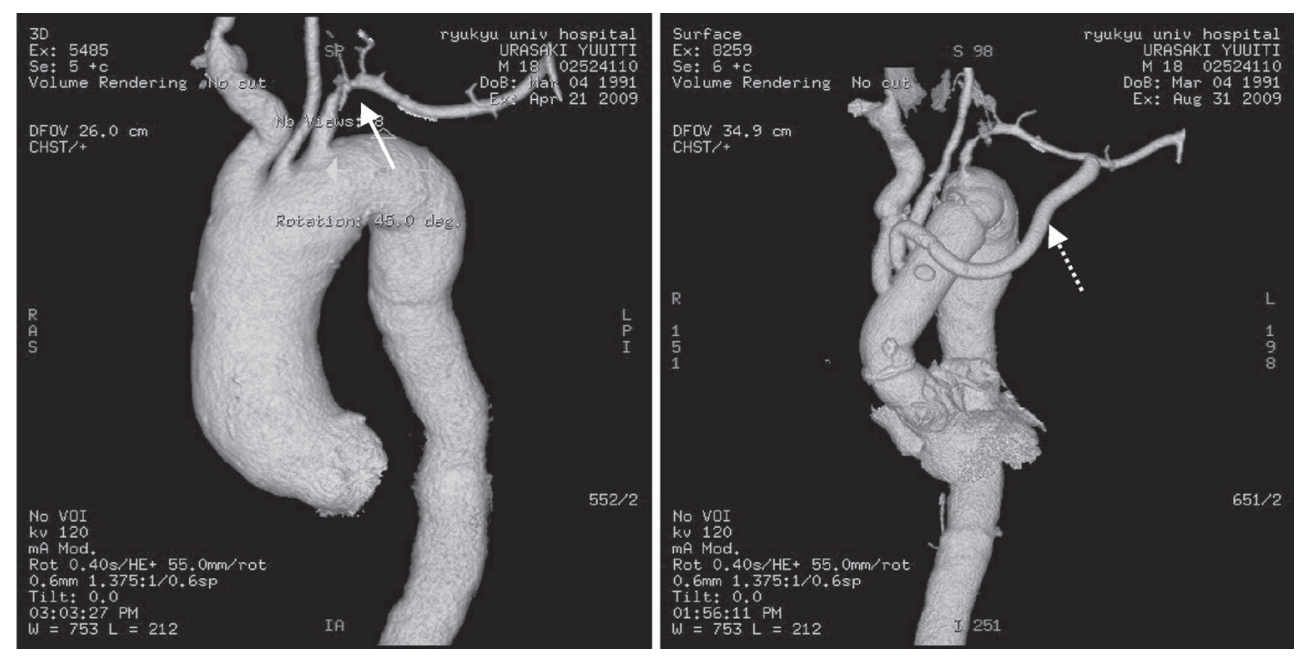

A $\mid$ B

Fig. 1 Preoperative and postoperative computed tomography (CT) images.

A) Preoperative CT image shows aneurysmal dilation from Sinus of Valsalva to arch aorta and stenosis of left subclavian artery (arrow).

B) Postoperative CT image shows good reconstruction of root and arch aorta with simultaneous reconstruction of left subclavian artery (dotted arrow).

graft harvested from the opposite femoral artery because of a suspected infectious pseudoaneurysm. Pathological assessment of a specimen of the right femoral artery included pseudoaneurysm revealed lymphocytic, but not neutrophilic infiltration. Internal medicine physicians suggested the possibility of collagen disease. However, he did not have a high fever, although the elevated CRP persisted even after repairing the pseudoaneurysm of the femoral artery. He was discharged 42 days after the operation despite having persistently high CRP values. Weekly chest computed tomography (CT) during conservative follow up imaging revealed no abnormal findings.

He was re-admitted with a high fever four months after the initial operation. Computed tomography at re-admission revealed a $2-\mathrm{cm}$ pseudoaneurysm at the posterior side of the aortic root (Fig. 2A and 2B), although CT did not recognize this pseudoaneurysm one month previously. The sagittal CT view revealed that the pseudoaneurysm originated from the left ventricular outflow or coronary button of the left coronary artery (Fig. 2C). Stenosis was found at the right femoral artery that had been repaired after the initial procedure using an allograft, and a pseudoaneurysm at the right axillary artery that had been cannulated. The preoperative course of this patient was also unusual. Initial laboratory findings at two months before the first operation indicated no inflammatory signs: WBC, 6100/mL; CRP, $0.5 \mathrm{mg} / \mathrm{dL}$. However, a high fever persisted for one week without leu- kocytosis, but CRP increased to $12.5 \mathrm{mg} / \mathrm{dL}$ after cardiac catheterization. We then evaluated the patient for collagen disease. Because antibiotics rapidly abolished the high fever, we investigated its etiology. Negative findings for leukocytosis and bacterial cultures ruled out endocarditis and infectious pseudoaneurysm. Serum procalcitonin was within the normal range. The high CRP level that was found before the initial procedure remained elevated $(5-10 \mathrm{mg} / \mathrm{dL})$ and erythrocyte sedimentation rate (ESR) was $105 \mathrm{~mm} / \mathrm{h}$. Component C3 and C4 levels were within normal limits and autoantibodies were negative. Therefore, we administered oral corticosteroid ( $40 \mathrm{mg} /$ day) to control the inflammation. Four days later, the fever subsided, and CRP recovered to within normal limits. We gradually decreased the corticosteroid to $20 \mathrm{mg}$ /day with reference to his CRP and ESR values. We operated on the pseudoaneurysm one month after starting corticosteroid administration. A laceration of about $1 \mathrm{~cm}$ transverse that surrounding thickness hard tissue seems to chronic inflammation was located below the left coronary cusp. We applied a modified Bentall procedure, including coronary artery reconstruction using the Piehler technique with a small prosthetic graft, because a severe connective tissue adhesion prevented harvesting of the coronary artery. We constructed an original composite graft with translocation of a mechanical valve to avoid repeated dehiscence of the anastomotic site. The artificial valve was sutured to the vascular prosthesis $1 \mathrm{~cm}$ from the edge. The composite 

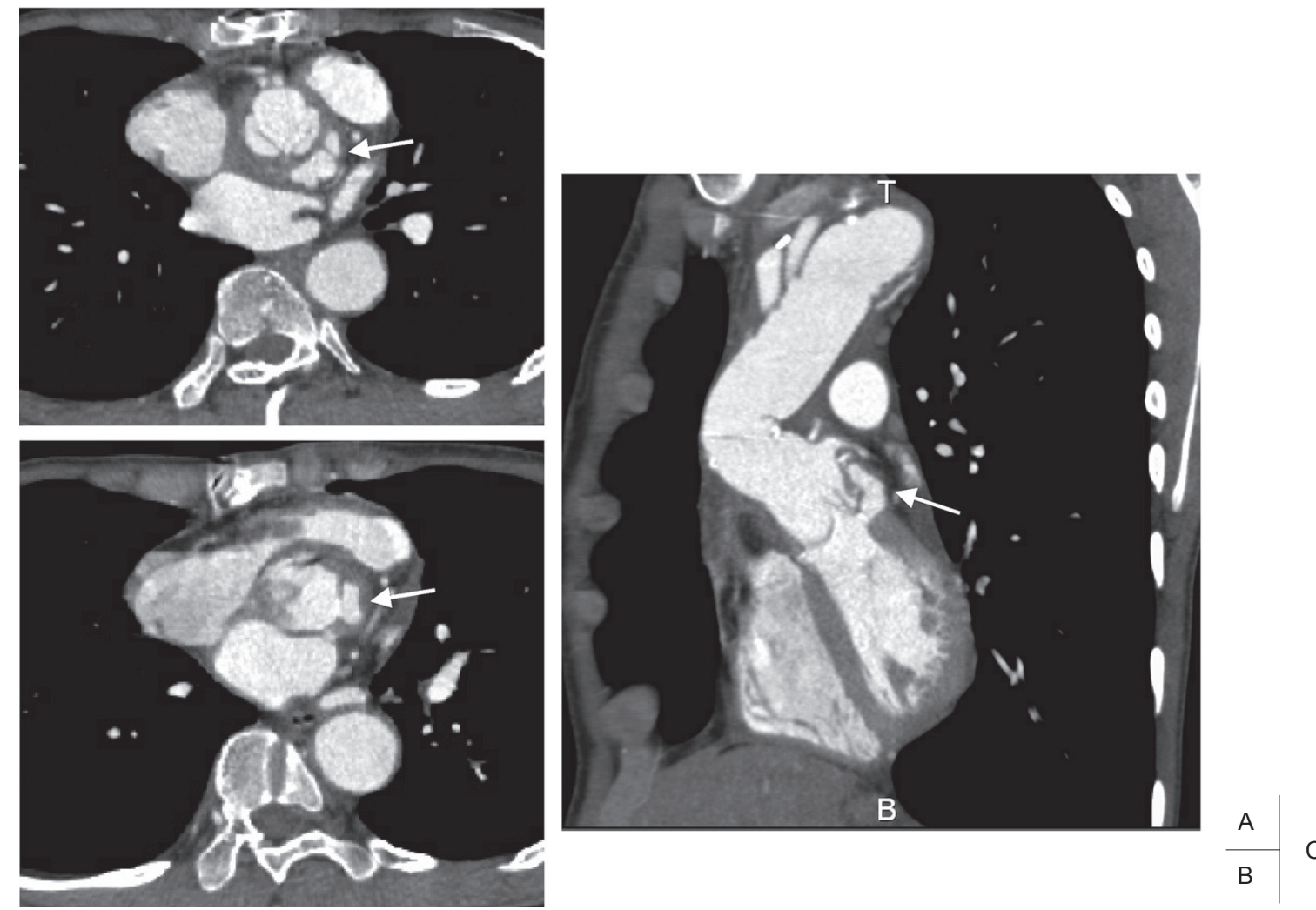

Fig. 2 Computed tomography (CT) images of pseudoaneurysm.

A and B) Images show pseudoaneurysm at posterior side of aortic root (arrow).

C) Sagittal CT view shows that pseudoaneurysm originated from left ventricular outflow.

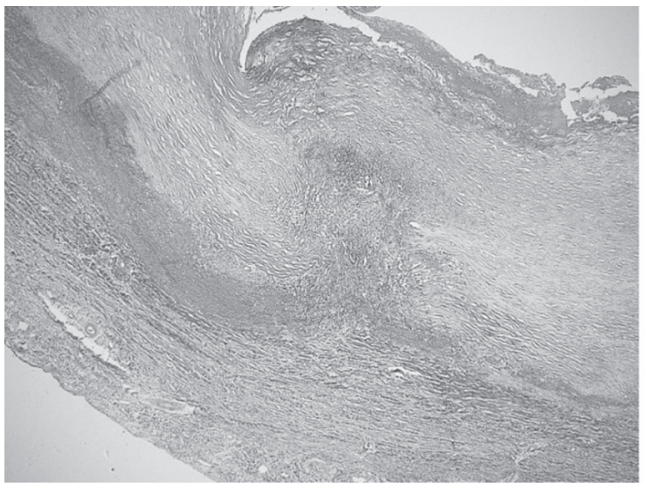

Fig. 3 Pathological findings of specimen.

A and B) Hematoxylin-eosin (HE) staining at $\times 40$ and $\times 100$ magnification, respectively

Lymphocytic infiltration is significant.

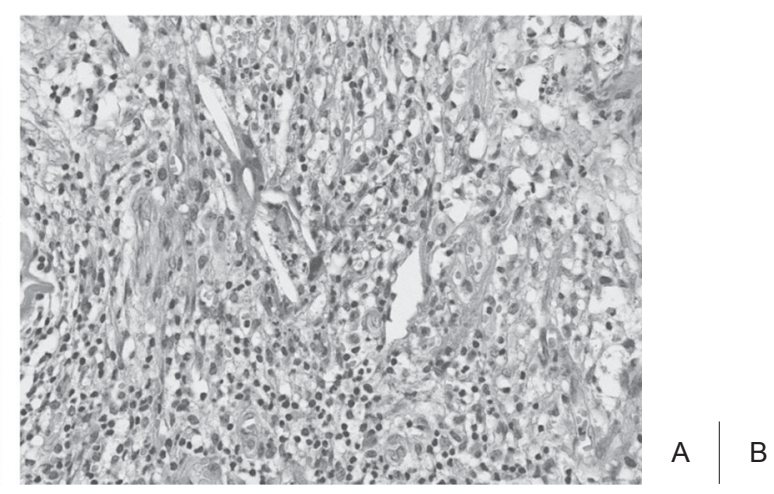

graft was implanted by suturing the vascular prosthesis with only Teflon felt strip to reinforce around the aortic annulus. Pathological assessment of a specimen of the aorta including annular tissue taken at second procedure revealed significant lymphocytic infiltration and thick, fibrous adventitia but no central medial necrosis (Fig. 3). The postoperative course was uneventful under corticosteroid administration. Postoperative CT revealed no abnormalities and he was discharged 21 days after this procedure.

\section{DiscuSSION}

Marfan syndrome is an autosomal dominant disorder 
of connective tissue involving the skeletal, ocular and cardiovascular systems ${ }^{5}$ ) that is often complicated by extended aortic aneurysms. Our patient had been diagnosed with Marfan syndrome at another hospital, and this was reconfirmed at the pediatric department of our hospital. His symptoms included typical skeletal signs such as being above average height $(180 \mathrm{~cm})$, having dolichostenomelia (elongated limbs), a thin, narrow face and dural ectasia. Moreover, his old twin brother had been diagnosed with Marfan syndrome. Therefore, we considered that he had annulo-aortic ectasia and an arch aneurysm associated with Marfan syndrome. We thus performed root reconstruction including valve sparing (David II) and standard total arch replacement. Preoperative CT identified stenosis of the left subclavian artery, and we, therefore, considered the underlying collagen disease.

Takayasu's arteritis is a nonspecific inflammatory disorder of unknown etiology, involving the aorta, its main branches, and pulmonary arteries, causing arterial stenosis/occlusion or dilation. The aortic root and ascending aorta are dilated in some patients with Takayasu's arteritis. ${ }^{6)}$ In our case, causing of aneurysm was not clearly identified to Takayasu's arteritis or Marfan syndrome, but the postoperative arterial events were nevertheless associated with active inflammation due to Takayasu's arteritis. Pathological assessment of a specimen of the aorta taken at the second procedure revealed no central medial necrosis, but significant lymphocytic infiltration and thick, fibrous adventitia indicating Takayasu's arteritis. 1) $^{\text {) }}$

The most serious complications after AVR or root reconstruction for Takayasu's arteritis are dehiscence of the anastomotic site and the formation of pseudoaneurysms. ${ }^{1-3)}$ Ando et al. ${ }^{6}$ reported a $4.6 \%$ (3 of 65 patients) incidence of prosthetic valve detachment after AVR among patients with Takayasu's arteritis. Shiono et al. ${ }^{2}$ reported that the potential risk for anastomotic dehiscence is much higher in patients with Takayasu's arteritis than in those with dystrophic aneurysms of Marfan syndrome, even during the inactive phase of inflammation. Suzuki et al. ${ }^{7)}$ emphasized the importance of reinforcing sutures around the aortic annulus and Carrel's button technique for reconstructing the coronary artery. They also recommended postoperative steroid therapy for active inflammation in patients with Takayasu's arteritis who have undergone several surgical procedures. Some authors also recommend perioperative adjunct steroid therapy to avoid serious anastomotic dehiscence. ${ }^{3,7)}$ The postoperative course of our patient was unusual. A high fever persisted despite the absence of an obvious, infectious focus, and serum CRP remained elevated despite negative bacterial cultures. These findings indicated active inflammation in Takayasu's arteritis. Pathological assessment of a specimen of the aorta taken at initial procedure did not reveal specific findings. It might be considered that we operated on the patient in chronic and convalescence period of Takayasu's arteritis and Takayasu's inflammation was activated postoperative period. Controlling the inflammation with corticosteroid immediately after the initial operation might have prevented the postoperative complications. We believe that the pseudoaneurysm was not caused by a technical procedural error because chest CT findings after the first operation were normal, and those at one month before re-admission did not recognize the pseudoaneurysm. The second postoperative course was uneventful when the patient was under corticosteroid administration. We considered that peri-operative steroid therapy for active inflammation in patients with Takayasu's arteritis who have undergone several surgical procedures is important. Matsuura et al. ${ }^{1)}$ described that active inflammation might be a predictor of pseudoaneurysm. We administered corticosteroid while monitoring the patient's laboratory data (CRP and ESR). He remains on $5 \mathrm{mg}$ /day of oral corticosteroid at 18 months after reoperation.

The translocated Bentall procedure might provide an alternative to composite graft detachment, ${ }^{3,6,7)}$ and might be helpful because it allows circumferential fixation outside the aortic wall and double fixation at the aortic annulus, and prosthetic valves do not apply direct pressure to the aortic annulus. Ando et al. ${ }^{6}$ ) noted that the pressure of valve function directly affects the rigid sewing ring in patients with AVR, which causes a higher detachment rate. We also constructed an original composite graft with translocation of a mechanical valve to avoid repeated dehiscence of the anastomotic site.

This patient was unique in terms of having simultaneous Takayasu's arteritis and cardiovascular manifestations of Marfan syndrome that were surgically treated. To our knowledge, this is a very rare example of concomitant Takayasu's arteritis and Marfan syndrome complicating a pseudoaneurysm after aortic root reconstruction. ${ }^{8)}$ Strict follow-up is required to prevent further anastomotic dehiscence.

\section{REFERENCES}

1) Matsuura K, Ogino H, Kobayashi J, et al. Surgical 
treatment of aortic regurgitation due to Takayasu arteritis: long-term morbidity and mortality. Circulation 2005; 112: 3707-12 Epub 2005 Dec 5. [Medline] [CrossRef]

2) Shiono M, Hata M, Sezai A, et al. Reoperation for ascending aortic aneurysm, coronary ostial aneurysm and patent Cabrol trick after bentall operation for aortitis syndrome. Circ J 2005; 69: 861-4. [Medline] [CrossRef]

3) Miyata T, Sato O, Deguchi J, et al. Anastomotic aneurysms after surgical treatment of Takayasu's arteritis: a 40 year experience. J Vasc Surg 1998; 27: 438-45. [Medline] [CrossRef]

4) Pyeritz RE, McKusick VA. The Marfan syndrome: diagnosis and management. N Engl J Med 1979; 300: 772-7. [Medline] [CrossRef]

5) Kalangos A, Baldovinos A, Beghetti M, et al. Ascend- ing aortic aneurysm associated with aortic insufficiency due to Takayasu's arteritis. Ann Thorac Surg 1999; 68: 248-50. [Medline] [CrossRef]

6) Ando M, Kosakai Y, Okita Y, et al. Surgical treatment of Behcet's disease involving aortic regurgitation. Ann Thorac Surg 1999; 68: 2136-40. [Medline] [CrossRef]

7) Stump DC, Califf RM, Topol EJ, et al. Pharmacodynamics of thrombolysis with recombinant tissue-type plasminogen activator. Correlation with characteristics of and clinical outcomes in patients with acute myocardial infarction. The TAMI Study Group. Circulation 1989; 80: 1222-30. [Medline] [CrossRef]

8) Kim KH, Lee C, Ahn H. Successful treatment in a patient with Takayasu's arteritis and Marfan syndrome. Ann Thorac Surg 2002; 74: 908-10. [Medline] [CrossRef] 